\title{
Control and improvement of antenna gain by using multilayer non-uniform metasurfaces
}

\author{
Jiaqi Han, Long $\mathrm{Li}^{*}$, Tianliang Zhang, and Rui Xi \\ Xidian University, Xi'an, Shaanxi, PR China
}

Received: 24 December 2017 / Accepted: 2 January 2019

\begin{abstract}
In this paper, a new high-gain antenna with beam control based on multilayer non-uniform metasurfaces (MNMSs) is proposed. The MNMS consists of multilayer non-uniform square and ring metal patches array. The phase-shift of the MNMS element can achieve $310^{\circ}$ with the variation of geometrical sizes. Moreover, four high-gain antennas based on MNMS element are designed, fabricated, and measured to realize $0^{\circ}$, $30^{\circ}, 45^{\circ}$, and $60^{\circ}$ beam control in pitching plane, respectively. Relative bandwidth of the proposed high-gain antenna is above $12 \%$. The simulated and measured results of the proposed antennas show that the wide-angle and azimuth direction beam control capability can be effectively realized by integrating different MNMS with the feeding horn antenna.
\end{abstract}

Keywords: Beam control / high-gain antenna / multilayer metasurfaces / metasurface

\section{Introduction}

Metasurface (MS) is a two-dimensional metamaterial structure, which is typically engineered by arranging a set of electrically small scatterers into a two-dimensional pattern at a surface or interface. In recent years, on the basis of the traditional multilayer frequency selective surface (FSS), a design method of achieving wideband MS by utilizing multilayer non-resonant structure is proposed [1-3]. As can be referred in [3], the miniaturization possesses the advantages of good frequency selection, angle stability, and a larger range of phase-shift. A good design of Huygens' MS for refraction of normally incident beam towards 71.8 degrees was presented in [4]. According to the theory of array antenna, the phase shifts of each unit of the MS need to be compensated to adjust and control the electromagnetic waves propagation direction. The theory of phase compensation [5-7] of the transmission array antenna is based on the compensation of the path transmission from the center of the feeding antenna [8-10]. Most previous studies mainly focused on steering electromagnetic waves using uniform MSs [11-16]. In this paper, we propose a non-uniform MS to control and improve the gain of a feeding antenna. A standard X-band horn antenna is used as a feeding source. According to the theory of array antenna, the main beam direction control and high gain antenna can be achieved by integrating multilayer non-uniform metasurfaces (MNMSs). In this

\footnotetext{
* Corresponding author: lilong@mail.xidian.edu.cn
}

paper, by varying the size of the square patch and the grid line to adjust the transmission phase of MNMS, the proposed high-gain antenna can realize a flexible beam control. The X-band high-gain antennas are implemented by using different MNMS, and the main beam with angles of $\theta=0^{\circ}, \theta=30^{\circ}, \theta=45^{\circ}$, and $\theta=60^{\circ}$ are realized, respectively. Also the beam control ability in azimuth plane is studied when the main beam is in a particular direction of $\theta=30^{\circ}$. Simulation and experiment results prove that the feeding horn antenna integrating with MNMS is a good method to achieve high gain and wideangle beam control.

\section{Antenna element}

In this paper, the MNMS unit is composed of non-uniform structures, which can be analyzed by using an effective circuit model and $\mathrm{J} / \mathrm{K}$ inverter. The technique is often used to analyze and synthesize a microwave filter and a multilayer FSS $[17,18]$. When the unit cell layer (order) increases, the bandwidth and the transmission phase-shift range will increase.

The MNMS unit is used to form a high-gain antenna that meets the purpose of beam control $[19,20]$. The phase compensation includes the phase delay caused by the different paths of electromagnetic wave from feed horn to each unit and phase difference of each unit for beam control. The way of phase compensation is by regulating MNMS unit size to adjust the transmission phase shift. For the proposed third-order unit, two parameters are chosen 


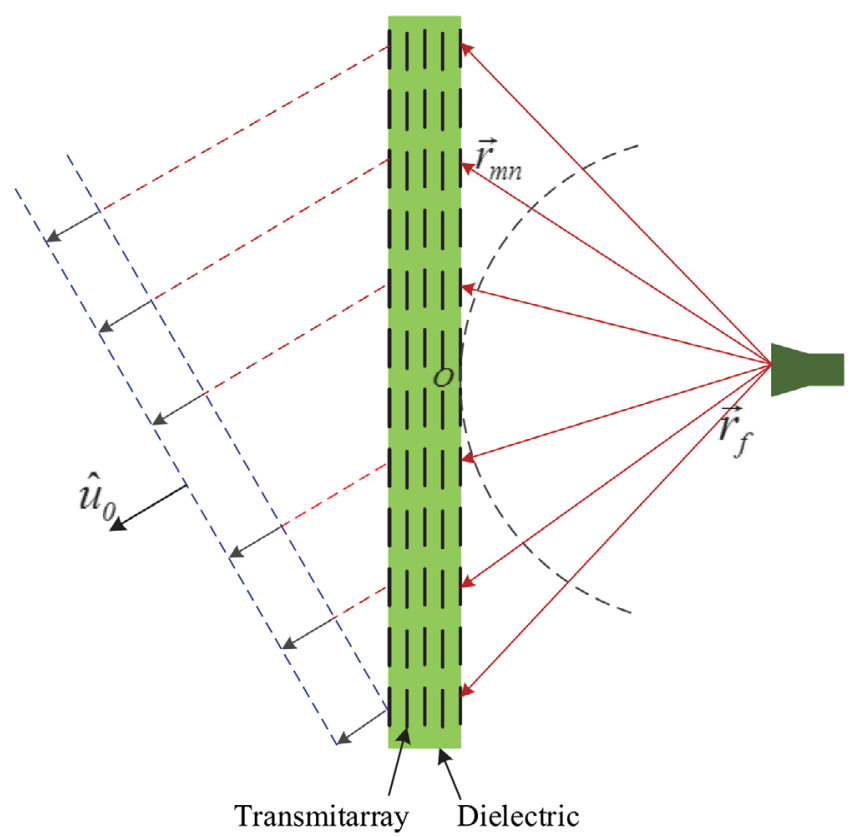

Fig. 1. The configuration of proposed high-gain antenna with MNMS.

as variables. These two parameters are the side length of the square metal patch $P$ and the width of the square metal ring $w$. In HFSS simulation software, the Floquet port combined with infinite period boundary condition is used to simulate periodic unit with different sizes and obtain the transmission phase characteristics. As shown in Figure 1, it is the configuration of the proposed high-gain antenna using MNMS. According to the array theory, sum of the radiation field of all elements in any direction will be expressed as follows [21-29]:

$$
\begin{aligned}
\vec{E}(\hat{u})= & \sum_{m=1}^{M} \sum_{n=1}^{N} \boldsymbol{F}\left(\vec{r}_{m n} \cdot \vec{r}_{f}\right) \boldsymbol{A}\left(\vec{r}_{m n} \cdot \hat{u}_{0}\right) \boldsymbol{A}\left(\hat{u}_{0} \cdot \hat{u}\right) \\
& \times \exp \left\{-j k_{0}\left[\left|\vec{r}_{m n}-\vec{r}_{f}\right|-\vec{r}_{m n} \cdot \hat{u}\right]+j \phi_{C}\right\}
\end{aligned}
$$

where $A$ represents the radiation pattern of unit cell, $F$ represents the radiation pattern of a feed, $\vec{r}_{m n}$ and $\vec{r}_{f}$ represent $m n$th radiating element position vector and the feed position vector, respectively. $\phi_{C}$ is the compensated phase of each unit, and $\hat{u}_{0}$ is the primary direction of the main beam $[15,19]$. The compensated transmission phase on each unit of MS is calculated from (1), according to the transmission phase, the corresponding size of unit can be obtained, which determines the overall structure of the MNMS.

Figure 2 shows the geometry of the MS unit cell, which is composed of a five-layer structure. Three layers are square metal patches, the other two layers are square metal rings. As shown in Figure 2a, the configuration is that each of square metal patch and square metal ring are in the form of interval arrangement. The metal structure is located in the middle of the dielectric substrate, and the thickness of each layer is $h=1.5 \mathrm{~mm}$. The dielectric constant of substrate is 2.65 with $\tan \delta=0.005$. Figure $2 \mathrm{~b}$ shows the (a)

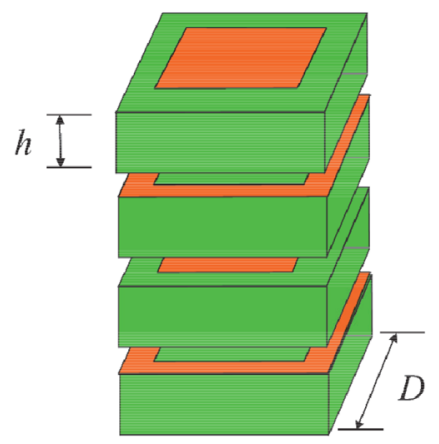

(b)

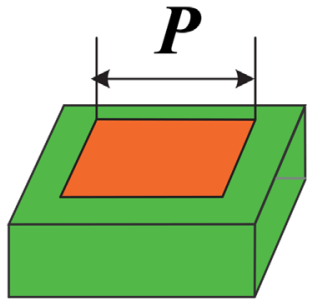

(c)

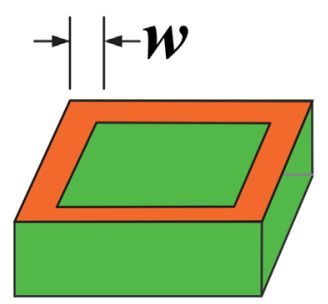

Fig. 2. Geometry structure of MNMS unit cell, (a) multilayer model, (b) square metal patch, and (c) square metal ring.

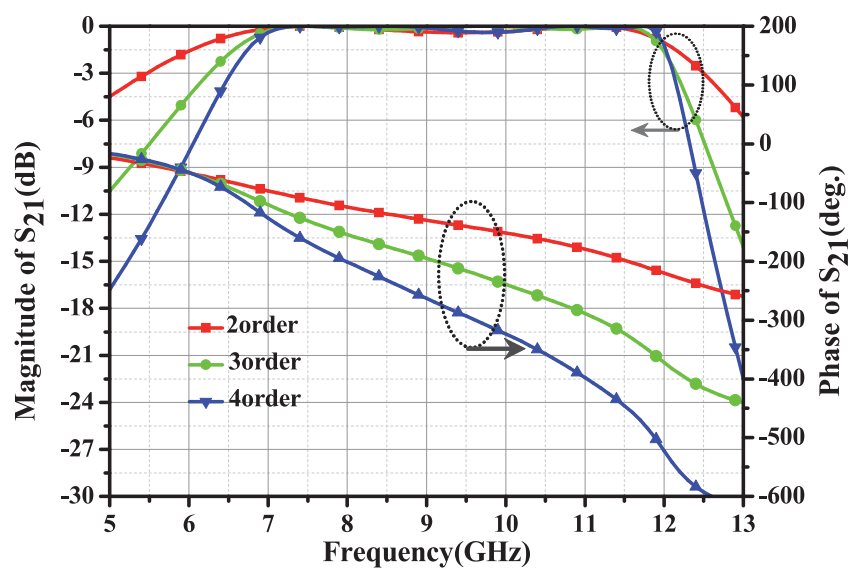

Fig. 3. The transmission magnitude and phase response curves of the second-order, third-order, and fourth-order model.

geometry of square metal patch with side length $P$. Figure 2c shows the square metal ring layer structure with metal ring width $w$. Period of this unit is $D=10 \mathrm{~mm}$ which is a subwavelength unit in X-band. It is useful for developing flexible beam control antenna.

The amplitude and phase response curves of the secondorder, third-order, and fourth-order model are illustrated in Figure 3, respectively. The $-3 \mathrm{~dB}$ bandwidths of the second-order, third-order, and fourth-order structures reduce in turn; however, the transmission phase ranges increase in turn in their $-3 \mathrm{~d} \mathrm{~B}$ passband. As seen from the Figure 3, the phase-shift range of the second-order structure within $-3 \mathrm{~dB}$ passband is about $200^{\circ}$, the phase-shift range of the third-order structure is about $310^{\circ}$, and the phase-shift range of the fourth order structure 
(a)

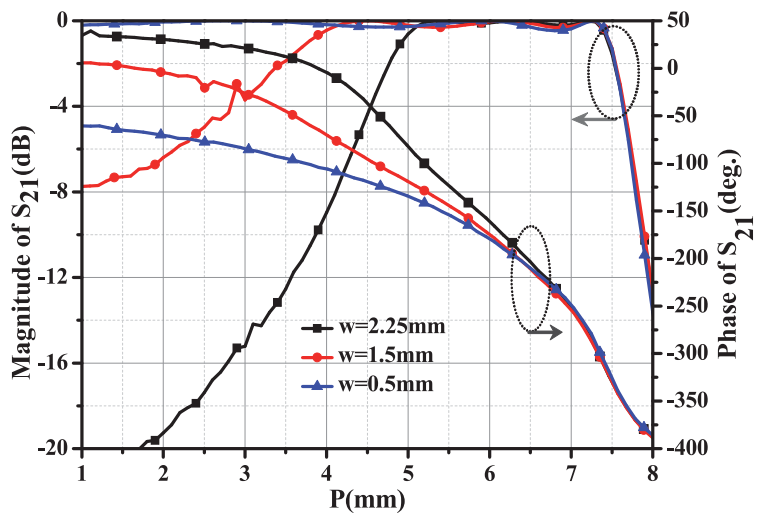

(b)

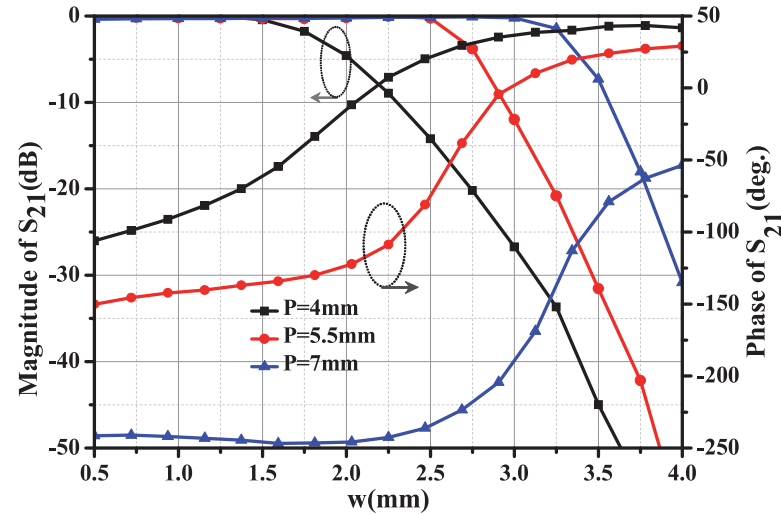

Fig. 4. The effect of size of MNMS unit on the transmission phase and magnitude of $S_{21}$, (a) $P$ and (b) $w$.

(a)

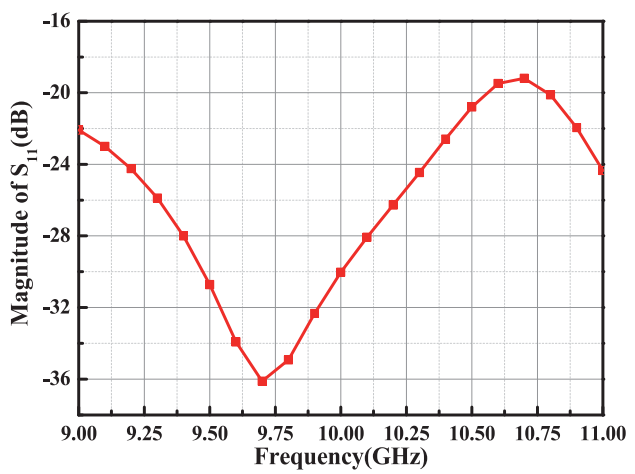

(b)

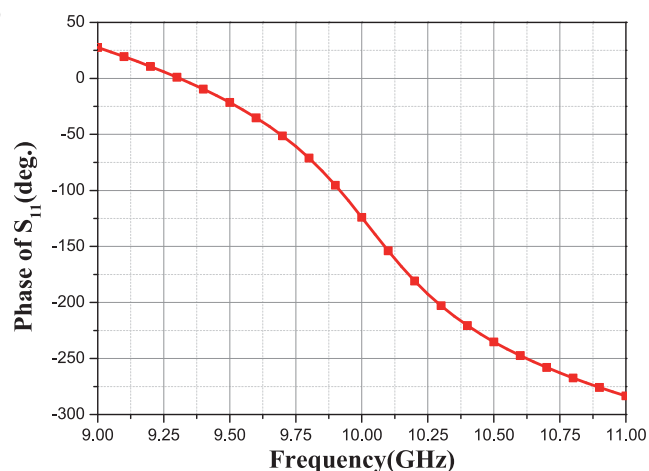

Fig. 5. The reflection coefficient of the MNMS unit, (a) the magnitude of $S_{11}$, (b) the phase of $S_{11}$.

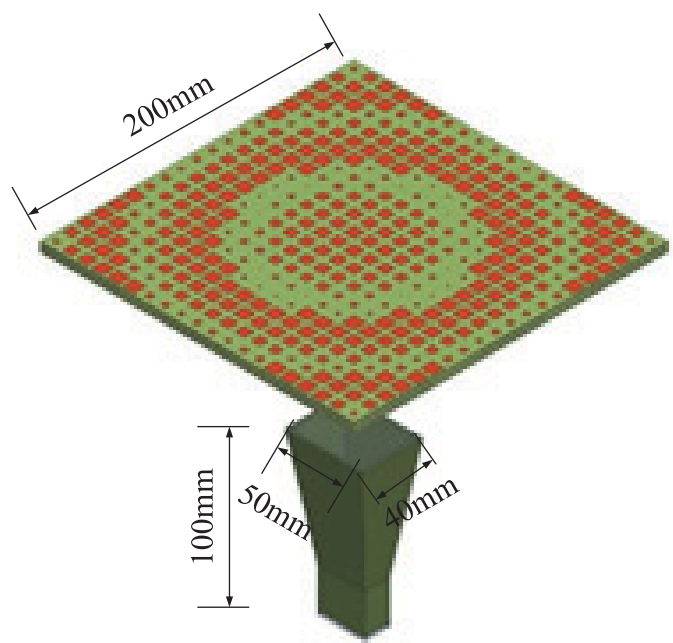

Fig. 6. Overall model of the high-gain antenna with MNMS.

is about $400^{\circ}$. The transmission phase characteristics are adjusted by changing the unit size. However, as the layer numbers of the MS increase, the phase sensitivity and the linearity will decrease, which is very important in the design of high-gain antenna and it affects the accuracy of the design. Therefore, the third-order element is selected to form the MNMS with a phase range of $310^{\circ}$ in this paper, which basically meets the design requirements of the beam control.

As shown in Figure 4, the side length of square metal patch $P$ and the width of square metal ring $w$ have an obvious impact on the phase and magnitude of transmission coefficient $S_{21}$ of the MNMS unit. The center working frequency is $f=10 \mathrm{GHz}$. It is shown that the side length of metal patch $P$ and the width of square metal ring $w$ impact on MNMS unit transmission phase and transmission coefficient. When selecting the range of the unit size parameter, one can make the changing of metal patches size $P$ more closely and the width of metal ring more sparsely. Finally, the transmission phase shift range for a relatively suitable transmission coefficient of $310^{\circ}$ is determined. Figure 5 is the reflection coefficient of the MNMS unit. The $S_{11}$ magnitude in the operating band is less than $-10 \mathrm{~dB}$, which indicates good working performance. The phase characteristic of the reflection coefficient is shown in Figure 5b, the reflection phase shift range of the unit in the operating band reaches $315^{\circ}$. It also corresponds to the phase shift range of the transmission coefficient of the third-order unit.

\section{High-gain antenna design and analysis}

As shown in Figure 6, the high-gain antenna is designed with $20 \times 20$ MNMS units, and a standard horn antenna working at X-band is utilized as the feed. The length of the horn antenna is $100 \mathrm{~mm}$, and the aperture size is $50 \mathrm{~mm} \times 40 \mathrm{~mm}$. The relative location of the MS and the feeding antenna is set as $F / D=0.5$, where the distance between the MNMS and horn antenna is set as $F$, and 
(a)

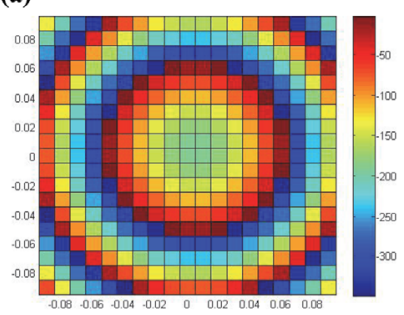

(b)

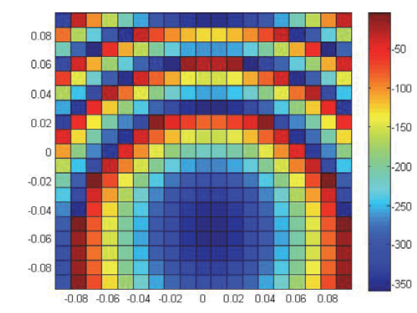

(c)

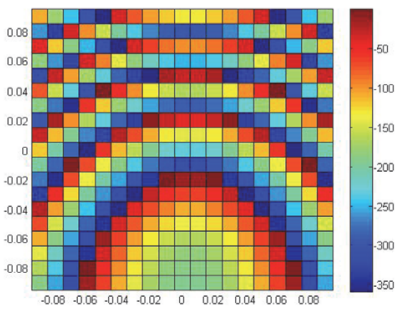

(d)

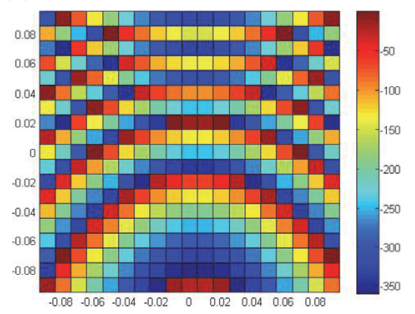

Fig. 7. The phase distribution diagram of main beam direction at (a) $\theta=0^{\circ}, \phi=0^{\circ}$, (b) $\theta=30^{\circ}, \phi=0^{\circ}$, (c) $\theta=45^{\circ}, \phi=0^{\circ}$, and (d) $\theta=60^{\circ}, \phi=0^{\circ}$, respectively.

(a)

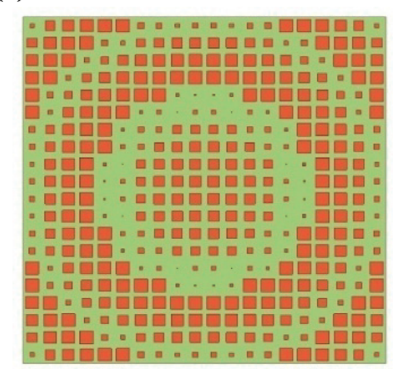

(c)

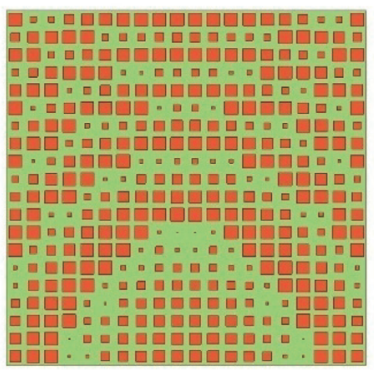

(b)

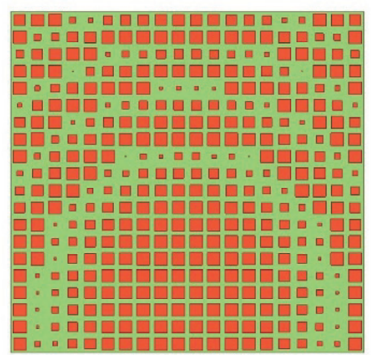

(d)

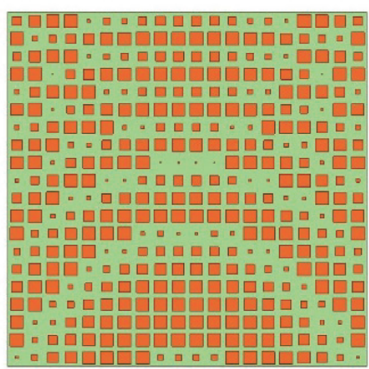

Fig. 8. Designed four high-gain antennas for different main beam directions at (a) $\theta=0^{\circ}, \phi=0^{\circ}$, (b) $\theta=30^{\circ}, \phi=0^{\circ}$, (c) $\theta=45^{\circ}$, $\phi=0^{\circ}$, and (d) $\theta=60^{\circ}, \phi=0^{\circ}$.

the dimension of the MNMS is set as $D$. The whole array size is $200 \mathrm{~mm} \times 200 \mathrm{~mm}$ with the $10 \mathrm{~mm}$ period of each MNMS unit, hence the distance between the MNMS and its source is $F=100 \mathrm{~mm}$.

Four MNMS structures are designed to achieve different deflection angles of $\theta=0^{\circ}, \theta=30^{\circ}, \theta=45^{\circ}$, and $\theta=60^{\circ}$, respectively. The detailed design procedure and characteristics of the four MSs are introduced as follows. According to the formula (1), the corresponding phase

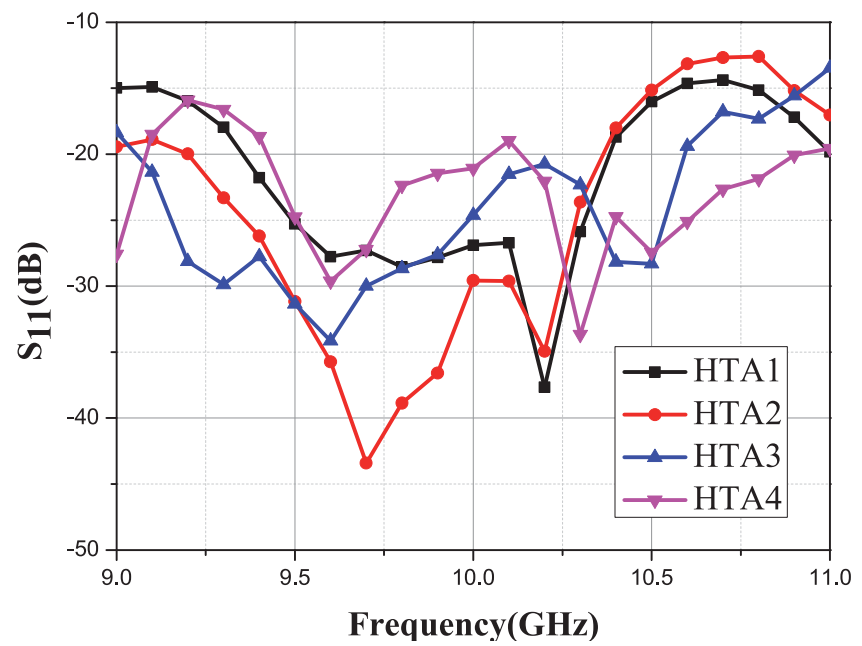

Fig. 9. The reflection coefficient of four high-gain antennas.

correction of each unit can be calculated and the corresponding phase compensation of the whole aperture is shown in Figure 7. There are four different phase distribution diagrams of the MNMS with the main beam directions of $\theta=0^{\circ}, \phi=0^{\circ} ; \theta=30^{\circ}, \phi=0^{\circ} ; \theta=45^{\circ}, \phi=0^{\circ}$; and $\theta=60^{\circ}, \phi=0^{\circ}$, respectively. Layouts of the four MSs are shown in Figure 8. It should be noting that the diagram is top view of the four MNMS structure. By comparing Figures 7 and 8 it can be seen that there is a clear correspondence between the compensation phase and its size.

The reflection coefficient of the four high-gain antennas with different MNMS is shown in Figure 9. The main beam of the antenna 1 (HTA1) is $\theta=0^{\circ}, \phi=0^{\circ}$, its $-3 \mathrm{~dB}$ gain bandwidth is from $9.17 \mathrm{GHz}$ to $11.7 \mathrm{GHz}$. And its relative bandwidth performance is $24.2 \%$. The main beam of the antenna 2 (HTA2) is pointed to the point of $\theta=30^{\circ}, \phi=0^{\circ}$, the $-3 \mathrm{~dB}$ gain bandwidth is from $9.0 \mathrm{GHz}$ to $10.5 \mathrm{GHz}$ with a $15.4 \%$ relative bandwidth. The main beam of the antenna 3 (HTA3) is $\theta=45^{\circ}, \phi=0^{\circ}$, and the gain bandwidth of $-3 \mathrm{~dB}$ is from $9.3 \mathrm{GHz}$ to $10.6 \mathrm{GHz}$ with the relative bandwidth of $13.7 \%$. Antenna 4 (HTA4) directs to $\theta=60^{\circ}, \phi=0^{\circ}$, the $-3 \mathrm{~dB}$ gain bandwidth is from $9.26 \mathrm{GHz}$ to $10.45 \mathrm{GHz}$, and its relative bandwidth is $12.1 \%$. From the above analysis, it can be seen that the four antennas with different MNMS have high gain performance within a wide frequency band. It is shown in Figure 10 that the comparison of gain patterns in the above half space among the four antennas and the feeding source horn antenna at $10 \mathrm{GHz}$ and $10.5 \mathrm{GHz}$. It can be seen from Figure 10a that from antenna 1 (HTA1) to antenna 4 (HTA4), the main beam can range from $0^{\circ}$ to $60^{\circ}$. Meanwhile, the MNMS converges the radiation pattern of feeding horn antenna and the gain of the horn antenna improves $11.4 \mathrm{~dB}, 10.2 \mathrm{~dB}, 9.0 \mathrm{~dB}$, and $7.4 \mathrm{~dB}$, respectively. Figure $10 \mathrm{~b}$ shows that the gain characteristics and the beam control performance can still be maintained when the antennas operate at $10.5 \mathrm{GHz}$. It can be seen from Figure 11 that all $-3 \mathrm{~dB}$ gain bandwidth of these four antennas is above $12 \%$. And the maximum 
(a)

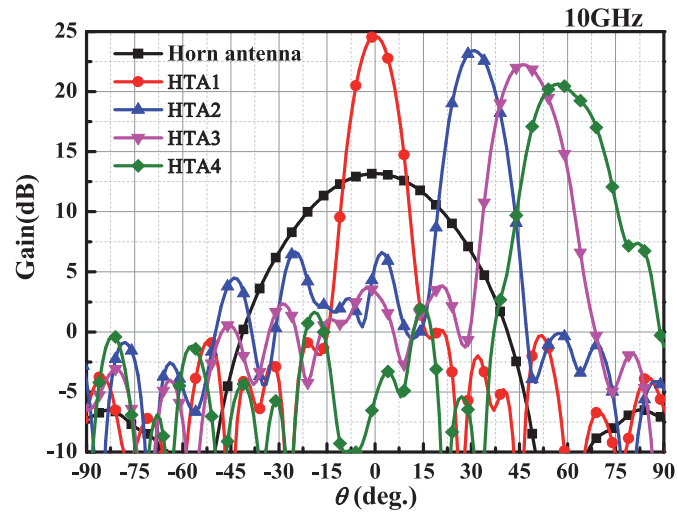

(b)

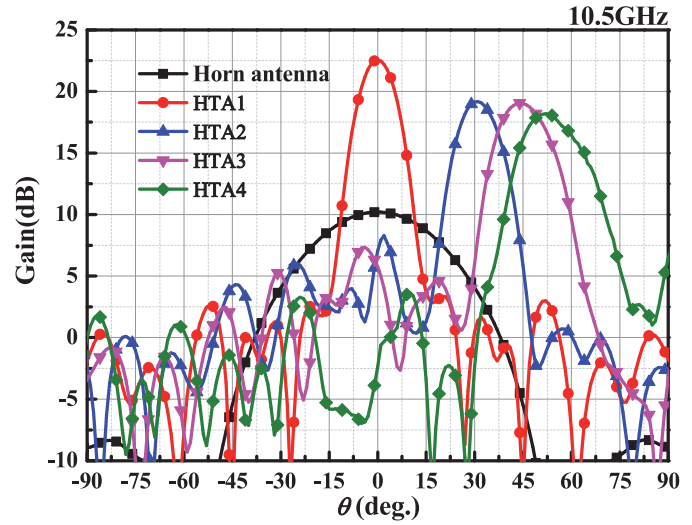

Fig. 10. Comparison of gain patterns among the four high-gain antennas and the feeding source horn antenna, (a) $10 \mathrm{GHz}$, and (b) $10.5 \mathrm{GHz}$.

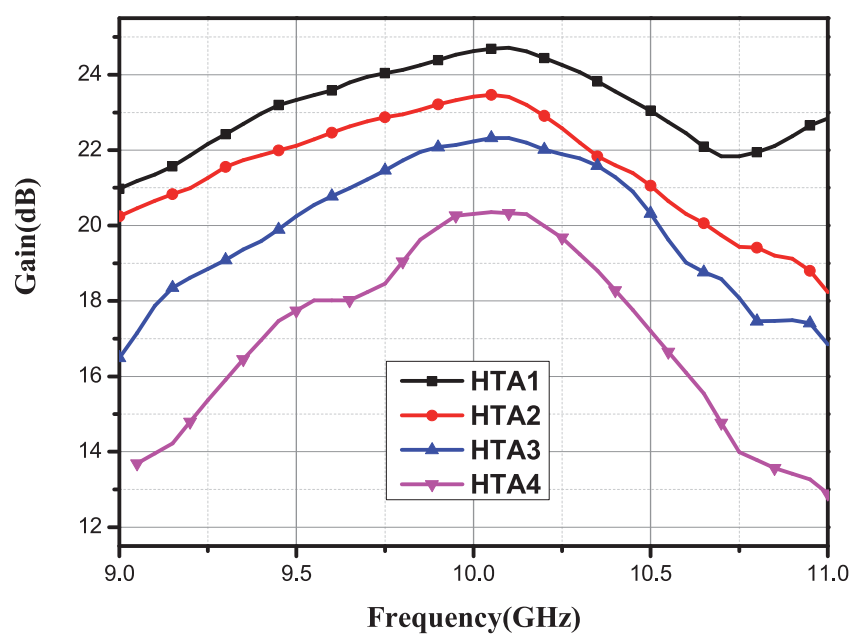

Fig. 11. Gain characteristics versus frequency of the four highgain antennas.

working bandwidth of HTA1 reaches $24.2 \%$. The analyses of the above characteristics prove that the use of nonresonant MNMS can realize wide beam control angle. Figure 12 shows the radiation efficiency of the antenna 1. It can be obtained from Figure 12 that the radiation

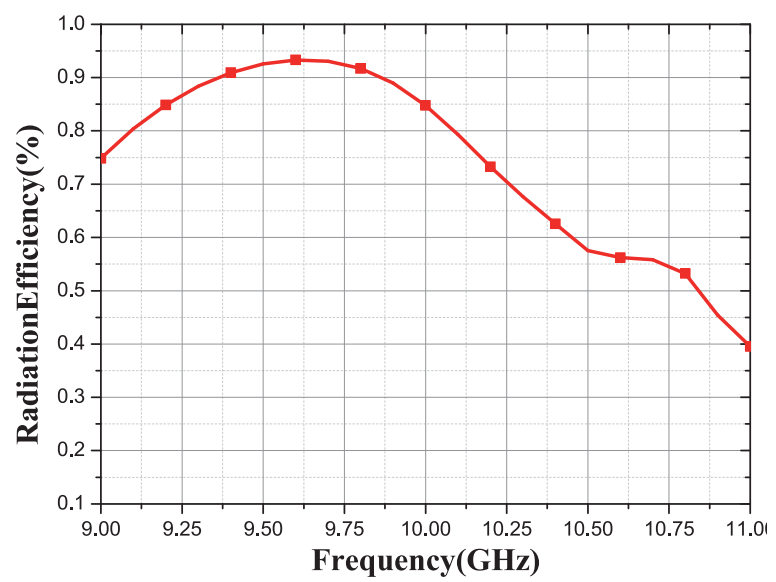

Fig. 12. The radiation efficiency of the antenna 1 . (a)

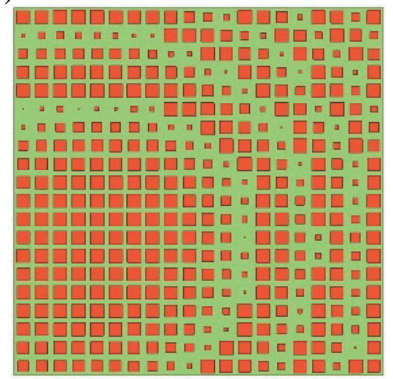

(b)

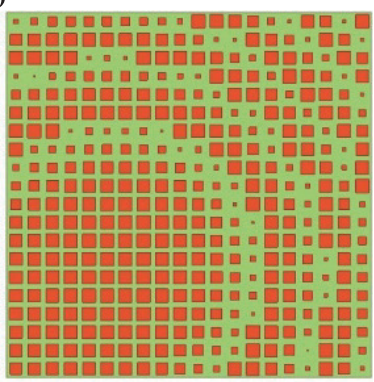

(c)

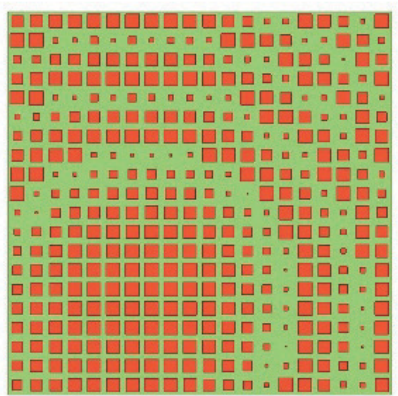

Fig. 13. Designed MNMS structure for different main beam directions at (a) $\theta=30^{\circ}, \phi=30^{\circ}$, (b) $\theta=30^{\circ}, \phi=45^{\circ}$, and (c) $\theta=30^{\circ}, \phi=60^{\circ}$.

efficiency between $9 \mathrm{GHz}$ and $10.25 \mathrm{GHz}$ is above $70 \%$, and the radiation efficiency at the operating frequency $10 \mathrm{GHz}$ is $85 \%$.

In order to analyze the controlling ability to azimuth angle of the proposed high-gain antenna, we design the polar angle at $\theta=30^{\circ}$ while setting the azimuth angles at $\phi=30^{\circ}, \phi=45^{\circ}$, and $\phi=60^{\circ}$, respectively. First of all, it should calculate the phase distribution of the array unit according to the formula (1), and use the corresponding relations between unit phase and unit size to design the MNMS structure, as shown in Figure 13. With the similar feeding horn antenna and MNMS aperture size, the radiation patterns are shown in Figure 14. 


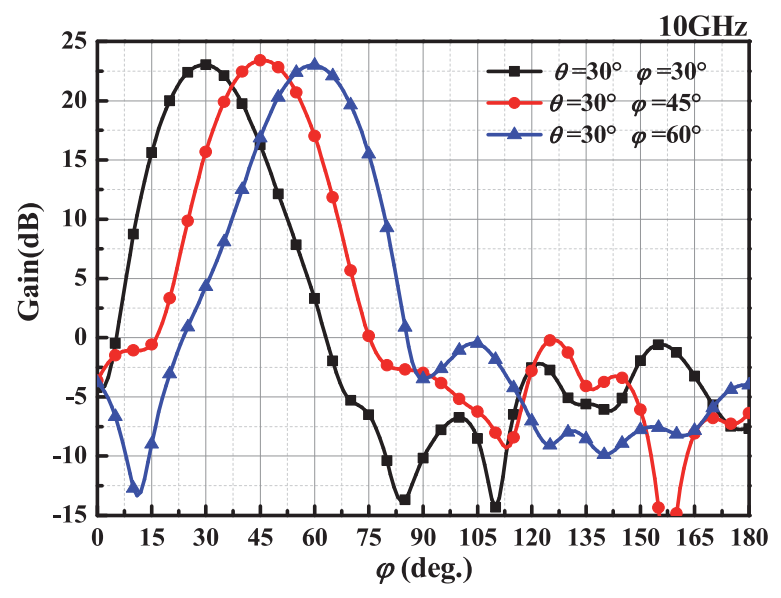

Fig. 14. The gain curve of different azimuth angle transmission antennas at $10 \mathrm{GHz}$.

(a)

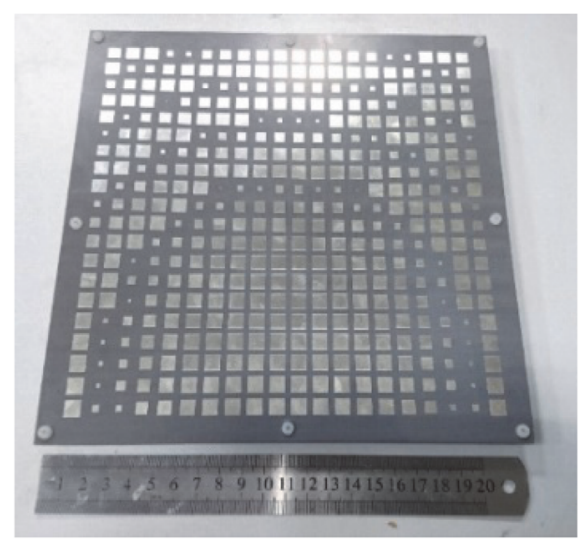

(b)

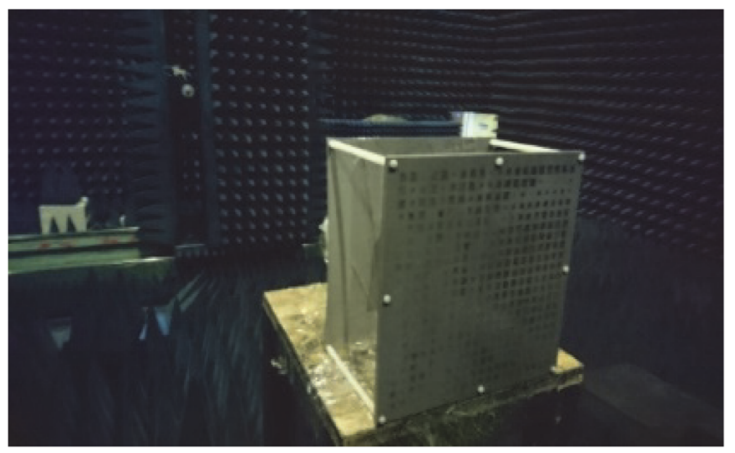

Fig. 15. (a) Top view of the antenna (HAT2), and (b) measurement environment for HTA2 in the microwave anechoic chamber.

It can be seen that when the pitch angle is confirmed at $\theta=30^{\circ}$, the control of the MNMS with azimuth angles at $\phi=30^{\circ}, \phi=45^{\circ}$, and $\phi=60^{\circ}$ also show good performance. The gain is almost the same with that of HTA2. It can be clearly seen from above figures that the main beam can be deflected in both the pitch angle and azimuth angle simultaneously. Therefore, it can realize the control and improvement of the feeding antenna by using MNMSs. (a)

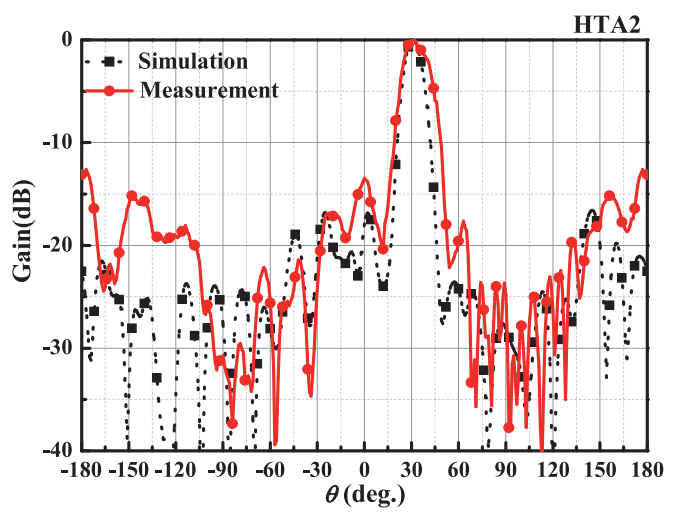

(b)

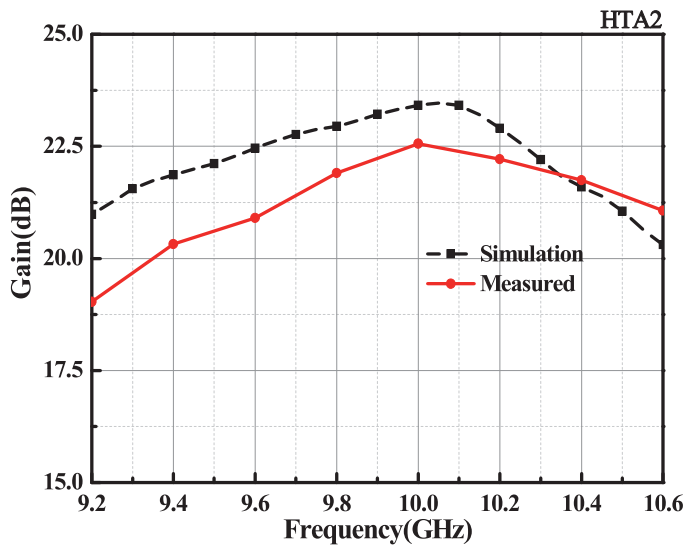

(c)

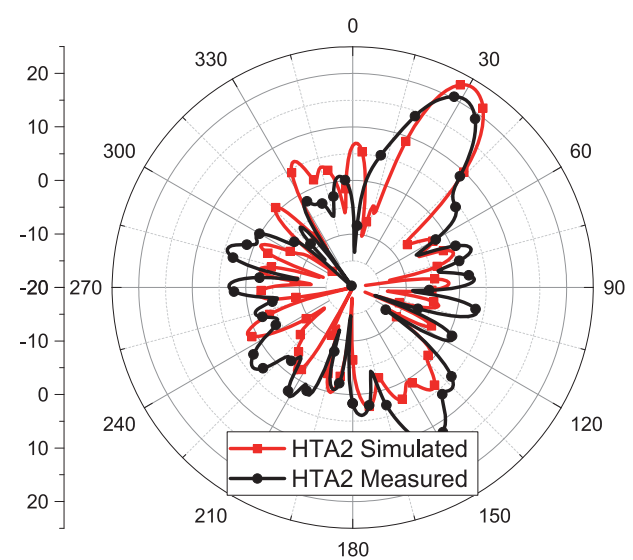

Fig. 16. Comparison of measured results and simulated result of HTA2, (a) normalized radiation pattern, (b) gain characteristics versus frequency, and (c) realized gain patterns.

\section{Experimental results and analysis}

In order to verify this method and design procedure, the antenna 2 (HTA2) is fabricated and measured. In the process of fabrication, we designed $10 \mathrm{~mm}$ margins for mounting holes. Figure $15 \mathrm{a}$ is the fabricated MNMS prototype, which is made up of four layer MSs and fixed with nylon screws. The antenna is measured in microwave anechoic chamber which is shown in Figure 15b. The measurement result of HTA2 is shown in Figure 16, which 
shows the gain characteristics at eight frequency points from $9.2 \mathrm{GHz}$ to $10.6 \mathrm{GHz}$. The comparison of normalized simulation and measurement radiation patterns at $10 \mathrm{GHz}$ is shown in Figure 16. It can be seen that the measured HTA2 results get a little worse compared to simulation ones. The main reason is due to the fabrication errors of the metal size and the air gap of the different layers; however, the measured and simulated beam control angles are basically consistent.

\section{Conclusion}

In this paper, a new high-gain antenna based on MNMSs is proposed. By utilizing the MNMS unit, different compensation phases are designed to direct the main beam to $0^{\circ}, 30^{\circ}, 45^{\circ}$, and $60^{\circ}$ in the pitch angles with high-gain. Moreover, it is analyzed that the beam ability of the feeding antenna composed of MNMS units in the azimuth, which can realize the main beam at three azimuth angles of $30^{\circ}, 45^{\circ}$, and $60^{\circ}$ which also achieve high gain characteristics. By testing the example of HTA2, the experimental results and the simulation results are in good agreement, which verifies the properties of high gain and beam deflection. The simulation and the measurement results of the proposed antenna show that it can realize the flexible improvement and control of feed beam radiation by employing MNMS.

This work is supported by National Key R\&D Program of China, and is supported by National Natural Science Foundation of China under Contract No.51477126, and supported by Technology Explorer and Innovation Research Project.

\section{References}

1. B.A. Munk, Frequency selective surfaces: theory and design (Wiley, New York, 2000)

2. A.H. Abdelrahman, A.Z. Elsherbeni, F. Yang et al., Transmission phase limit of multilayer frequency-selective surface for transmitarray designs, IEEE Trans. Antennas Propag. 62, 690 (2014)

3. Y. Zhang, R. Mittra, W. Hong, A zoned two-layer flat lens design, International Workshop on Antenna Technology (iWAT2011), March 2011, pp. 412-415

4. M. Chen, E. Abdo-Sanchez, A. Epstein, G.V. Eleftheriades, Theory, design, and experimental verification of a reflectionless bianisotropic Huygens' metasurface for wide-angle refraction, Phys. Rev. B 97, 125433 (2018)

5. M.A. Al-Joumayly, N. Behdad, Wideband planar microwave lenses using sub-wavelength spatial phase shifters, IEEE Trans. Antennas Propag. 59, 4542 (2011)

6. C.G.M. Ryan, M.R. Chaharmir, J. Shaker et al., A wideband transmitarray using dual-resonant double square rings, IEEE Trans. Antennas Propag. 58, 1486 (2010)

7. M. Sazegar, Y. Zhegn, C. Kohler et al., Beam steering transmitarray using tunable frequency selective surface with integrated ferroelectric varactors, IEEE Trans. Antennas Propag. 60, 5690 (2012)
8. L. Wang, X. Yin, S. Li et al., Phase corrected substrate integrated waveguide $\mathrm{H}$-plane horn antenna with embedded metal-via arrays, IEEE Trans. Antennas Propag. 62, 1854 (2014)

9. D. Ramaccia, F. Scattone, F. Bilotti et al., Broadband compact horn antennas by using EPS-ENZ metamaterial lens, IEEE Trans. Antennas Propag. 61, 2929 (2013)

10. D. Ramaccia, M. Barbuto, A. Monti et al., Exploiting intrinsic dispersion of metamaterials for designing broadband aperture antennas: theory and experimental verification, IEEE Trans. Antennas Propag. 64, 1141 (2016)

11. H. Nokano, S. Mitsui, J. Yamauchi, Tilted-beam high gain antenna system composed of a patch antenna and periodically arrayed loops, IEEE Trans. Antennas Propag. 62, 2917 (2014)

12. A.K. Layer, G.V. Eleftheriades, A multilayer negativerefractive-index transmission-line (NRI-TL) metamaterial free-space lens at X-band, IEEE Trans. Antennas Propag. 55, 2746 (2007)

13. L. Meng, M. Behdad, Wideband true-time-delay microwave lenses based on metallo-dielectric and all-dielectric lowpass frequency selective surfaces, IEEE Trans. Antennas Propag. 61, 4109 (2013)

14. Y.J. Lee, J. Yeo, R. Mittra et al., Thin frequency selective surfaces (FSS) superstrate with different periodicities for dual-band directivity enhancement, IEEE International Workshop on Antenna Technology: Small Antennas and Novel Metamaterial, March 2005

15. G.V. Trentini, Partially reflecting sheet arrays, IRE Trans. Antennas Propag. 4, 666 (1956)

16. D.M. Pozar, S.D. Targonski, H.D. Syrigos, Design of millimeter wave micropatch reflectarrays, IEEE Trans. Antennas Propag. 45, 287 (1997)

17. Y. Li, L. Li, Y. Zhang, C. Zhao, Design and synthesis of multilayer frequency selective surface based on antennafilter-antenna using Minkowski fractal structures, IEEE Trans. Antennas Propag. 63, 133 (2015)

18. T. Zhang, L. Li, R. Xi, Design of a multilayer FSS transmission high-gain antenna array and analysis of the beam control, Asia-Pacific Conference on Antennas and Propagation APCAP'2017, October 2017, Xi'an, China, pp. 285-287

19. J. Huang, J.A. Encinar, Reflectarray antennas (Wiley-IEEE Press, United States of America, 2008)

20. Y. Hao, A.H. Alomainy, C.G. Parini, Antenna-beam shaping from offset defects in UC-EBG cavities, Microw. Opt. Technol. Lett. 43, 108 (2004)

21. H. Moghadas, M. Daneshmand, P. Mousavi, Half-phasegradient partially reflective surface for a reconfigurable dualbeam scanning cavity antenna, Antennas and Propagation on Society International Symposium, July 2012, pp. 1-2

22. H. Kaouach, L. Dussopt, J. Lanteri et al., Wideband low-loss linear and circular polarization transmitarrays in V-band, IEEE Trans. Antennas Propag. 59, 2513 (2011)

23. C. Pfeiffer, A. Grbic, Millimeter-wave transmitarrays for wavefront and polarization control, IEEE Trans. Antennas Propag. 61, 4407 (2013)

24. H. Hasani, M. Kamyab, A. Mirkamali, Broadband reflectarray antenna incorporating disk elements with attached phasedelay lines, IEEE Antennas Wirel. Propag. Lett. 9, 156 (2010)

25. E. Almajali, D. Mcnamara, J. Shaker et al., Derivation and validation of the basic design equations for symmetric subreflectarrays, IEEE Trans. Antennas Propag. 60, 2336 (2012) 
26. D.C. Chang, M.C. Huang, Multiple-polarization micropatch reflectarray antenna with high efficiency and low crosspolarization, IEEE Trans. Antennas Propag. 43, 829 (1995)

27. Y. Li, M.E. Bialkowski, A.M. Abbosh, Single layer reflectarray with circular rings and open-circuited stubs for wideband operation, IEEE Trans. Antennas Propag. 60, 4183 (2012)
28. C. Han, C. Rodenbeck, K. Chang et al., A C/Ka dual frequency dual layer circularly polarized reflectarray antenna with micropatch ring elements, IEEE Trans. Antennas Propag. 52, 2871 (2004)

29. W. Hu, M. Arrebola, R. Cahill, $94 \mathrm{GHz}$ dual-reflector antenna with reflectarray subreflector, IEEE Trans. Antennas Propag. 57, 3043 (2009)

Cite this article as: Jiaqi Han, Long Li, Tianliang Zhang, Rui Xi, Control and improvement of antenna gain by using multilayer non-uniform metasurfaces, EPJ Appl. Metamat. 6, 4 (2019) 